\title{
Optimization of the preparation of aqueous suspensions of waxy maize starch nanocrystals using a response surface methodology
}

Hélène Angellier ${ }^{1}$, Luc Choisnard ${ }^{2}$, Sonia Molina-Boisseau ${ }^{1}$, Patrick Ozil ${ }^{3}$, Alain Dufresne ${ }^{4}$

${ }^{1}$ Centre de Recherches sur les Macromolécules Végétales (CERMAV-CNRS), Université Joseph Fourier, BP 53, 38041 Grenoble cedex 9, France.

2 Département de Pharmacologie Moléculaire (DPM), Université Joseph Fourier, 38243 Meylan Cedex, France

${ }^{3}$ Laboratoire d'Electrochimie et de Physico-chimie des Matériaux et des Interfaces (LEPMIINPG), BP 75, 38402 St Martin d'Hères Cedex, France.

4 Ecole Française de Papeterie et des Industries Graphiques de Grenoble (EFPG-INPG), BP 65, 38402 St Martin d'Hères Cedex, France.

\begin{abstract}
Response surface methodology was used to investigate the effect of five selected factors on the selective $\mathrm{H}_{2} \mathrm{SO}_{4}$ hydrolysis of waxy maize starch granules. These predictors were temperature, acid concentration, starch concentration, hydrolysis duration and stirring speed. The goal of this study was to optimize the preparation of aqueous suspensions of starch nanocrystals, i.e. to determine the operative conditions leading to the smallest size of insoluble hydrolyzed residue within the shortest time and with the highest yield. Therefore empirical models were elaborated for the hydrolysis yield and the size of the insoluble residues using a Central Composite Face Design (CCFD) involving 31 trials. They allowed us to show that it was possible to obtain starch nanocrystals after only 5 days of $\mathrm{H}_{2} \mathrm{SO}_{4}$ hydrolysis with a yield of $15 \mathrm{wt} \%$ and having the same shape as those obtained from the classical procedure after 40 days of $\mathrm{HCl}$ treatment, with a yield of $0.5 \mathrm{wt} \%$.
\end{abstract}

Keywords : Design of experiments (DOE); waxy maize starch; acid hydrolysis; nanocrystals

* corresponding author: Alain.Dufresne@efpg.inpg.fr 


\section{Introduction}

There is currently a considerable interest in processing polymeric composite materials filled with nanosized rigid particles (essentially inorganic). This class of material attracting both scientific and industrial communities is called "nanocomposites". Because of the nanometric size effect, these composites have some unique outstanding properties with respect to their conventional microcomposite counterparts. Nowadays the application of nanoparticles and then the development of new nanocomposite materials are restricted by both their limited availability and their strong tendency to aggregate preventing their homogeneous dispersion within a continuous matrix, which is the key step required for high mechanical performances.

Starch is a natural polymer available in large amounts from several renewable plant sources and it is produced in abundance beyond available markets. Starch is the cheapest biopolymer and is totally biodegradable. These two main reasons lead to the growing interest in the non-food usage of starch-based products for applications in which synthetic polymers have traditionally been the materials of choice. It is well known that native starch granules contain more or less concentric "growth rings" that are readily visible by optical or electron microscopy. ${ }^{1}$ Acid treatment is needed to reveal the concentric lamellar structure of starch granules. It has been shown that these lamellae, around $5000 \AA$ thick, have subspacing of a few hundred $\AA .^{2}$ The purpose of this treatment using hydrochloric acid is to dissolve away regions of low lateral order so that the water-insoluble, highly crystalline residue may be converted into a stable suspension by subsequent vigorous mechanical shearing action. ${ }^{3}$

In previous works ${ }^{4,5}$ such starch nanocrystals obtained from potato starch granules were used as a reinforcing phase in a polymeric matrix and displayed substantially improved mechanical properties. The insoluble hydrolyzed residue obtained from waxy maize was found to be composed of crystalline nanoplatelets around 5-7 nm thick with a length of 20-40 nm and a width 
of $15-30 \mathrm{~nm}^{6}$ However, the main drawbacks for the more extensive use of such nanoplatelets in nanocomposite applications are the duration (40 days of treatment) and the yield (0.5 wt\%) of the $\mathrm{HCl}$ hydrolysis step. $^{3}$

Starch disruption by acid hydrolysis depends on many factors such as the botanic origin, ${ }^{7-13}$ namely the crystalline type, the relative proportion of amylose and amylopectin, and the granules morphology. It also depends on the conditions of acid hydrolysis, namely the acid type, ${ }^{14,15}$ acid concentration, $^{7}$ temperature ${ }^{7}$ and hydrolysis duration. ${ }^{7}$ No deep interest has been brought to the influence of stirring and starch concentration. Suspensions were usually stirred manually every day and a large panel of starch concentration has been used, for instance $1.5 \mathrm{wt} \%{ }^{10}$ $1.67 \mathrm{wt} \%,{ }^{16,17} 2 \mathrm{wt} \%,{ }^{13} 2.5 \mathrm{wt} \%,{ }^{12} 5 \mathrm{wt} \%{ }^{3,4,6,9}$ and $33 \mathrm{wt} \% .^{18}$ If $\mathrm{HCl}$ hydrolysis has been largely studied, ${ }^{7}$ equivalent works about sulfuric hydrolysis remain limited.

It is now accepted that the partial crystallinity of native starch granules is due to a clustered organization of amylopectin side chains ${ }^{16}$ and that an increasing amylose content results in a decrease of the susceptibility of starch granule to acidic degradation. ${ }^{19}$ Therefore amylopectinrich starch, i.e. waxy maize, was chosen for the present study. Furthermore, a previous work ${ }^{20}$ has shown that $\mathrm{H}_{2} \mathrm{SO}_{4}$ acid hydrolysis resulted in more stable suspensions than hydrochloric ones, which is an important characteristic for composite materials processing. Thereby, we chose to focus this work on optimizing the $\mathrm{H}_{2} \mathrm{SO}_{4}$ hydrolysis of waxy maize starch granules taking into account the following parameters: hydrolysis duration, temperature, acid concentration, starch concentration and stirring speed.

Response surface methodology was carried out to investigate the effect of these selected factors. This classical method is largely used and well adapted to process optimization in the macromolecular science area. ${ }^{21-23}$ Laser granulometry, yield calculation and transmission electron microscopy were used to characterize the insoluble hydrolysis residues. 


\section{Experimental}

Acid hydrolysis. A given weight of native waxy maize starch granules (Waxylis ${ }^{\mathrm{TM}}$, Roquette S.A.) was mixed with $250 \mathrm{~mL}$ of $\mathrm{H}_{2} \mathrm{SO}_{4}$ solution at a known concentration in a $500 \mathrm{~mL}$ erlenmeyer. The suspensions were then put on a plat-form in a thermo-stated atmosphere and continuously stirred at a selected speed with an orbital shaking action. After various durations of hydrolysis, the suspensions were washed by successive centrifugations in distilled water until neutrality. They were stored at $4^{\circ} \mathrm{C}$ with several drops of chloroform.

The hydrolysis yield (wt\%) was calculated as the ratio between the weight of freeze-dried hydrolyzed particles and the initial weight of native granules for an aliquot of $50 \mathrm{~mL}$ taken in the $250 \mathrm{~mL}$ of hydrolyzed suspensions. It was verified that these aliquots were representative of the entire volume of $250 \mathrm{~mL}$.

Characterization. Transmission electron microscopy (TEM) observations were performed using a Philips CM200 microscope with a $80 \mathrm{kV}$ accelerating voltage.

Laser granulometry measurements were carried out with a Malvern Mastersizer. The suspensions were characterized from the median particle size $\mathrm{d}_{50}$, which divides the population into two equal halves.

Design of experiments. The five following parameters were varied:

- hydrolysis temperature $\mathrm{u}_{1}$,

- acid concentration $\mathrm{u}_{2}$,

- initial starch concentration $\mathrm{u}_{3}$,

- hydrolysis duration $\mathrm{u}_{4}$,

- speed of stirring, $\mathrm{u}_{5}$. 
Parameters $\mathrm{u}_{3}$ and $\mathrm{u}_{5}$ do not influence hydrolysis kinetic because of its catalytic nature. These two parameters have been however taken into account in the present work because they were varying in a large range in the literature.

The settings of the different parameters were determined for all the predictors from our preliminary experiments ${ }^{20}$ and keeping in mind the two following intuitive rules. On the one hand, the optimal conditions have a lot of chance to be outside of the region of interest if selecting a too narrow variation range. On the other hand, the predictive power of the model risks to be poor if choosing a too large range. Table 1 shows the minimal ( $\left.\mathrm{u}_{\mathrm{i}} \mathrm{min}\right)$, the midrange ( $\left.\mathrm{u}_{\mathrm{i}} \mathrm{mid}\right)$ and the maximal ( $\left.\mathrm{u}_{\mathrm{i}} \max \right)$ values used for each parameter, which respectively correspond to $-1,0$ and +1 levels in terms of orthogonal variable $\mathrm{x}_{\mathrm{i}}$ defined as:

$$
x_{i}=\frac{2 \cdot\left(u_{i}-u_{i} \min \right)}{\left(u_{i} \max -u_{i} \min \right)}
$$

Table 1.

Here the two responses under study were:

- The hydrolysis yield as previously defined, yyield (wt $\%)$,

- the median size $\mathrm{y}_{\text {size }}(\mu \mathrm{m})$ of hydrolyzed particles treated by Ultra Turrax $(13,000 \mathrm{rpm}$, concentration of starch of $1 \mathrm{wt} \%$, treated volume of $60 \mathrm{~mL}$ ) during $5 \mathrm{~min}$ measured by laser granulometry.

Since both response non linearities and interactions between factors were expected, the response model was postulated to be a quadratic one for each response $\eta_{k}$. This model may be expressed in terms of orthogonal variables as:

$$
\eta_{k}=b_{0}+\sum_{i=1}^{5} b_{i} x_{i}+\sum_{\substack{i, j=1 \\ i \neq j}}^{5} b_{i j} x_{i} x_{j}+\sum_{i=1}^{5} b_{i i} x_{i}^{2}
$$


This postulated model takes into account the linear effects $\left(\mathrm{b}_{\mathrm{i}} \mathrm{x}_{\mathrm{i}}\right)$ and the quadratic ones $\left(b_{\mathrm{ii}} \mathrm{x}_{\mathrm{i}}^{2}\right)$ as well as first-order interactions $\left(\mathrm{b}_{\mathrm{ij}} \mathrm{x}_{\mathrm{i}} \mathrm{x}_{\mathrm{j}}\right)$ and its knowledge requires to estimate the coefficients $b_{i}, b_{i i}, b_{i j}$.

Experiments were conducted adopting a Central Composite Face Design (CCFD). This type of design was suitable for our objective, which was the optimization of a potential complex process. The CCFD (Table 2) involves a fractional factorial design $2^{5-1}$ (trials 1 to 16), the face centers (trials 17 to 26 - all coordinates equal to zero except one equal to +1 or -1 )) and five replications at the central point (trials 27 to 31 - all coordinates equal to zero). The run order of trials was randomized in order to prevent systematic errors.

The determination of the optimal conditions proposed by the MODDE software is based on the maximization of a desirability coefficient $\mathrm{D}$ defined as a weighted average of the individual response desirabilities $\mathrm{d}_{\mathrm{i}}::^{24}$

$$
D=\frac{1}{M} \sum_{i=1}^{M} d_{i}=\frac{1}{M} \sum_{i=1}^{M} w_{i}\left(\frac{y_{i}-T_{i}}{T_{i}-L_{i}}\right)^{2}
$$

$\mathrm{M}$ being the number of responses and the experimenter having to provide the following data for each response $\mathrm{y}_{\mathrm{i}}$ : the weight $\mathrm{w}_{\mathrm{i}}$ (here, $\mathrm{w}_{\mathrm{i}}=1$ for all the responses), the desired response target $\mathrm{T}_{\mathrm{i}}$ and its worst acceptable value $\mathrm{L}_{\mathrm{i}}$.

\section{Results and discussion}

Models and analysis. The responses measured for each trial are reported in Table 2.

Table 2.

Plotting the N-probability versus deleted studentized residuals has shown that there were no deviating experiments also called outliers, considering that the action limit was \pm 4 standard deviations (not shown in this paper). Thereby, no experiment was performed again nor excluded 
from the analysis. Moreover the analysis of raw data through the Box-Cox transformation showed that no response transformation was useful to improve the models, considering a 0.95 confidence interval.

The responses were fitted owing to a multi-linear regression method (MLR) as quadratic models expressed in terms of orthogonal variables $\mathrm{x}_{\mathrm{i}}$ as said above and refined using a backward step by step technique based on a 0,95 confidence level. ${ }^{25,26}$

The final refined models $\left(\eta_{\text {yield }}\right.$ and $\eta_{\text {size }}$ ) and their corresponding statistics (coefficient of regression $R^{2}$, coefficient of determination $R^{2}$ adj and coefficient of prediction $Q^{2}$ ) are as follow:

$$
\begin{aligned}
& \eta_{\text {yield }}=30.1615-7.7444 x_{1}-12.3722 x_{2}+1.7889 x_{3}-17.1111 x_{4}-2.3833 x_{5} \\
& +2.5625 x_{3} x_{5}+7.6051 x_{4}{ }^{2} \\
& \mathrm{R}^{2}=0.940, \mathrm{R}_{\text {adj }}^{2}=0.922, \mathrm{Q}^{2}=0.884 \\
& \eta_{\text {size }}=8.3223-0.9905 x_{1}-1.3572 x_{2}-0.1022 x_{3}-2.6844 x_{4}-0.6372 x_{5} \\
& +0.5712 x_{3} x_{5}+1.3166 x_{4}^{2} \\
& \mathrm{R}^{2}=0.859, \mathrm{R}_{\text {adj }}^{2}=0.817, \mathrm{Q}^{2}=0.751
\end{aligned}
$$

For both models, $\mathrm{R}^{2}, \mathrm{R}^{2}$ adj and $\mathrm{Q}^{2}$ statistics have quite high values close to unity with a difference $\mathrm{R}^{2}$ and $\mathrm{Q}^{2}$ lower than 0.2 , which is an indication of suitable models. Here the adjusted $\mathrm{R}^{2}$ statistics are greater than 0.85 , so proving a good descriptive power of the models. This power can be illustrated besides by the plot of experimental responses versus the predicted ones (Figure 1) which should be ideally the diagonal line and is here characterized by an acceptable dispersion. Moreover the predictive power, as evidenced by $\mathrm{Q}^{2}$ values greater than 0.5 , is excellent for $\eta_{\text {yield }}$ and not so good for $\eta_{\text {size }}\left(Q^{2}=0.751\right)$ but still acceptable. ${ }^{21}$

Figure 1. 
Moreover a classical analysis of variance (ANOVA) using F-tests allows to analyze the total response variation by identifying the parts corresponding to the sources of variation (regression model, pure experimental error) and to analyze the residuals in order to point out the possible lack of fit of the postulated model when replicates are available. Here we could conclude that there is no lack of fit for the model concerning the hydrolysis yield while some lack of fit exists for the median size. That is however not critical because of a $\mathrm{Q}^{2}$ statistic greater than 0.5 $\left(Q^{2}=0.751\right)$.

The stirring speed $\left(\mathrm{x}_{5}\right)$ and overall the starch concentration $\left(\mathrm{x}_{3}\right)$ do not have any significant influence on the yield and the median size (Figure 2). Effectively, the probabilities of significance $(p)$ of estimated coefficients $b_{3}$ and $b_{5}$ were superior to the critical limit of 0.05 . They were respectively $\mathrm{p}=0.1598$ and $\mathrm{p}=0.0653$ for $\eta_{\text {yield }}$, and $\mathrm{p}=0.7230$ and $\mathrm{p}=0.0356$ for $\eta_{\text {size }}$. However, these terms were kept in models according to the hierarchy principle. It is quite surprising that starch concentration did not have an effect on the yield of hydrolysis and the median size of insoluble residues. We could have thought that an increase of starch concentration would have slowed down the diffusion of acid in the suspension. Starch concentration did not have an effect on the yield of hydrolysis and the median size of insoluble residues. We could have thought that a decrease of starch concentration would have favored the action of the catalyst. This result proves that, in the chosen ranges, acid is largely in excess in the reactor.

Only one interaction between starch concentration and stirring has been detected.

Figure 2.

The response surfaces and the corresponding contour plots were drawn for both responses. They allow illustrating the impact of two selected parameters by keeping the other three parameters at constant values (Figures 3 to 6 ). 
Figures 3 to 6.

In order to study the reliability of the models and validate them, several experimental conditions were tested. We succeeded in producing controlled yields with a respective confidence level of 0.95 . On the other hand, the experimental value of the median size was outside the predicted interval (difference between the measured sizes and the interval of prediction and the predicted values of $6.1 \%$ and $24 \%$, respectively) so confirming a light lack of fit for the model. Nevertheless the model will be used for optimizing the median size because of the suitable value of the $\mathrm{Q}^{2}$ statistic greater than 0.5 .

Optimization. The multivariable models obtained from the statically design of experiments were used for predicting the optimal conditions of $\mathrm{H}_{2} \mathrm{SO}_{4}$ hydrolysis that should allow obtaining aqueous suspensions of waxy maize starch nanocrystals in the shortest time, with the smallest median size particle and the highest yield.

We assumed that the highest yield for the preparation of starch nanocrystal suspensions that could be reached was about $30-40 \%$ which is the relative crystallinity of native waxy maize starch granules. ${ }^{27}$ Preliminary studies ${ }^{20}$ have shown that the morphology of nanocrystals began to be observed after 7 days of acid hydrolysis with a corresponding yield of 26.9 wt $\%$ and that 40 days of $\mathrm{HCl}$ hydrolysis were needed to obtain a suspension of insoluble residues all having the shape of nanoplatelets, with a corresponding yield of $0.5 \mathrm{wt} \%$. These results showed that selective acid hydrolysis takes time. Thereby, a reasonable goal was to obtain such suspensions in less than 7 days, with a yield of $20 \mathrm{wt} \%$.

For optimization, the yield and the median size were considered as targets, with a constrained time value and all other factors varying freely in the considered experimental domain (Table 3$)$. First, a target of $20 \mathrm{wt} \%$ for the yield $\left(\eta_{\text {yield }}\right)$ and of $5 \mu \mathrm{m}$ for the median size $\left(\eta_{\text {size }}\right)$ 
have been chosen, with a duration of hydrolysis of 7 days. These criteria corresponded to "optimization 1". Secondly, duration of hydrolysis of 5 days has been chosen, with the same target for the yield but a median size of $6 \mu \mathrm{m}$, considering that we would not have been able to obtain the same median size than in 7 days. These criteria corresponded to "optimization 2".

Table 3.

The sets of conditions proposed by the MODDE software are given in Table 4. The given $\log \mathrm{D}$ value is negative or near zero indicating that we are doing rather well.

Table 4.

Achieving an accuracy of 4 decimals for experimental conditions settings was of course impossible. Run 1 was performed using $35.35 \mathrm{~g}$ of starch $(14.14 \mathrm{wt} \%)$ mixed in $250 \mathrm{~mL}$ of $2.87 \mathrm{M} \mathrm{H}_{2} \mathrm{SO}_{4}$ solution (161.8 $\mathrm{mL}$ of $\mathrm{H}_{2} \mathrm{SO}_{4} 95 \%$ and $838.2 \mathrm{~mL}$ of distilled water), at $40^{\circ} \mathrm{C}, 100$ rpm and during 7 days. For run 2, $36.725 \mathrm{~g}$ of starch (14.69 wt\%) were mixed in $250 \mathrm{~mL}$ of $3.16 \mathrm{M} \mathrm{H}_{2} \mathrm{SO}_{4}$ solution ( $178.1 \mathrm{~mL}$ of $\mathrm{H}_{2} \mathrm{SO}_{4} 95 \%$ and $821.9 \mathrm{~mL}$ of distilled water), at $40^{\circ} \mathrm{C}, 100$ rpm and during 5 days.

The predictions calculated with a confidence interval of 0.95 and the measured responses corresponding to these sets of conditions are given Table 5 .

Table 5 .

Measured yields were inside the predictive intervals but not the median sizes, which were smaller than the lower limits. Whereas it confirmed the lack of prediction of the model $\eta_{\text {size }}$, this result is pleasantly surprising and interesting for our goal which was to prepare small residues, among others. Furthermore, contrary to all expectations, a smaller median size was obtained with the same yield after 5 days than after 7 days of acid treatment. Observations by transmission electron microscopy (TEM) were performed to verify the shape of the insoluble residues. 


\section{Characterization of optimized suspension of nanocrystals.}

Figure 7

Observations by TEM clearly shown that the insoluble residue obtained after 5 days of

optimized $\mathrm{H}_{2} \mathrm{SO}_{4}$ hydrolysis (Table 5) have the shape of parallelepiped nanoplatelets (Figure 7a). Nanoplatelets were generally observed in aggregates of 1 to $5 \mu \mathrm{m}$ (Figure 7a) or at best in barrets of several platelets (Figure 7b). Even if the parallelepipedic shape was the general shape that we observed, a lot of varying organizations (Figures 7b-d) were distinguished. Few stacks of nanoplatelets oriented edge-on were observed in a very little proportion (not shown here), what let assume that the platelets were well separated.

These observations lead to conclude that the optimization process was successful. We have shown that it was possible to obtain starch nanocrystals after 5 days of $\mathrm{H}_{2} \mathrm{SO}_{4}$ hydrolysis with a yield of $15 \mathrm{wt} \%$ having the same shape than those obtained after 40 days of $\mathrm{HCl}$ treatment, with a yield of $0.5 \mathrm{wt} \%$.

\section{Conclusion}

The statistical experimental design and the multi-linear regression analysis used in this study have proven to be very useful for establishing predictive models for both the yield of $\mathrm{H}_{2} \mathrm{SO}_{4}$ hydrolysis of waxy maize starch granules and the median size of insoluble residues after acid treatment. We achieved to produce aqueous suspensions of starch nanocrystals after 5 days of $3.16 \mathrm{M} \mathrm{H}_{2} \mathrm{SO}_{4}$ hydrolysis at $40^{\circ} \mathrm{C}, 100 \mathrm{rpm}$ and with a starch concentration of $14.69 \mathrm{wt} \%$ with a yield of $15.7 \mathrm{wt} \%$ and the same shape than those obtained after 40 days of $\mathrm{HCl}$ hydrolysis. It should allow considering preparation of starch nanocrystals for nanocomposite applications. 


\section{Acknowledgements}

The authors are grateful to Roquette (Lestrem, France) for the supply of waxy maize starch. Special thanks are given to Dr J.L. Putaux (CERMAV-CNRS) for his support in TEM observations and the discussions about acid hydrolysis.

\section{References}

(1) Nikuni, Z.; Whistler, R.L. J. Biochem. 1957, 44, 227.

(2) Frey-Wyssling, A.; Muhlethaler, K. in Ultrastructural Plant Cytology, Elsevier, The Netherlands, 1965.

(3) Battista, O.A., In Microcrystal Polymer Science, McGraw-Hill Book Company, 1975, 138.

(4) Dufresne, A.; Cavaillé, J.Y.; Helbert, W. Macromolecules 1996, 29, 7624.

(5) Dufresne, A.; Cavaillé, J.Y. J. Polym. Sci., Part B: Polym. Phys. 1998, 36, 2211.

(6) Putaux, J.L.; Molina-Boisseau, S.; Momaur, T.; Dufresne A. Biomacromolecules 2003, 4(5), 1198.

(7) Robin, J.P. PhD Thesis, Université Pierre et Marie Curie, Paris, France (1976).

(8) Duprat, F.; Gallant, D.; Guilbot, A.; Mercier, C.; Robin, J.P. In Les polymères végétaux, Ed. Bernard Monties, 176-231 (1980).

(9) Jane, J.; Wong, K.; McPherson, A. Carbohydr. Res. 1997, 300, 219.

(10) Jenkins, P.J.; Donald, A.M. Starch/Stärke 1997, 49, 262.

(11) Franco, C.M.L.; Ciacco, C. F.; Tavares, D.Q. Starch/Stärke 1998, 5, 193.

(12) Jayakody, L.; Hoover, R. Food Research International 2002, 35, 665.

(13) Gerard, C.; Colonna, P.; Buléon, A.; Planchot, V. Carbohydr. Polym. 2002, 48, 131.

(14) Muhr, A.H.; Blanshard, J.M.V.; Bates, D.R. Carbohydr. Polym. 1984, 4, 399. 
(15) Nägeli, C.W. Annalen der Chemie 1874, 173, 218.

(16) Robin, J.P.; Mercier, C.; Charbonnière, R.; Guilbot, A. Cereal Chem. 1974, 51, 389.

(17) Lintner, C.J. J. Prak. Chem. 1886 34, 378.

(18) Singh, V.; Ali, S.Z. Carbohydr. Polym. 2000, 41, 191.

(19) Planchot, V. PhD Thesis, Université de Nantes, France (1993).

(20) Angellier, H.; Molina-Boisseau, S.; Putaux, J.L.; Dupeyre, D.; Dufresne, A. Macromol. Symp., submitted.

(21) Karlsson, A.; Albertsson, A.-C. Biomacromolecules 2002, 3, 850.

(22) Behravesh, E.; Timmer, M.D.; Lemoine, J.J.; Liebschner, M.A.K.; Mikos, A.G. Biomacromolecules 2002, 3, 1263.

(23) Behravesh, E.; Jo, S.; Zygourakis, K.; Mikos, A.G. Biomacromolecules 2002, 3, 374.

(24) Eriksson, L.; Johansson, E.; Kettaneh-Wold, N.; Wikström, C.; Wold, S. In Design of experiments, principles and applications, Umetrics Academy, 2000.

(25) Goupy, J. In Plans d'expériences pour surfaces de réponse, Ed. Dunod, Paris, 1999.

(26) Darlington, R.B. In Regression and linear models, New York, Mc Grow Hill, 1990.

(27) Zobel, H.F. Starch/Stärke 1988, 40(1), 1. 
Table 1: Setting levels of parameters $u_{i}$

\begin{tabular}{lcccc}
\hline \multicolumn{1}{c}{ Parameter } & Unit & $\begin{array}{c}\text { Low level } \\
x_{i}=-1\end{array}$ & $\begin{array}{c}\text { Medium level } \\
x_{i}=0\end{array}$ & $\begin{array}{c}\text { High level } \\
x_{i}=+1\end{array}$ \\
\hline $\mathrm{u}_{1}$, temperature & ${ }^{\circ} \mathrm{C}$ & 35 & 37.5 & 40 \\
$\mathrm{u}_{2}$, acid concentration & $\mathrm{mol} \cdot \mathrm{L}^{-1}$ & 2.2 & 2.8 & 3.4 \\
$\mathrm{u}_{3}$, starch concentration & $\mathrm{g} / 100 \mathrm{~mL}$ & 5 & 10 & 15 \\
$\mathrm{u}_{4}$, time & day & 1 & 5 & 9 \\
$\mathrm{u}_{5}$, stirring & $\mathrm{rpm}$ & 0 & 50 & 100 \\
\hline
\end{tabular}


Table 2: Results obtained for the complete set of 31 experimental points

\begin{tabular}{|c|c|c|c|c|c|c|c|}
\hline Trial & $\mathrm{X}_{1}$ & $X_{2}$ & $X_{3}$ & $X_{4}$ & $X_{5}$ & $\begin{array}{c}Y_{\text {yield }} \\
(\%)\end{array}$ & $\begin{array}{l}Y_{\text {size }} \\
(\mu \mathrm{m})\end{array}$ \\
\hline 1 & -1 & -1 & -1 & -1 & +1 & 76.3 & 13.40 \\
\hline 2 & +1 & -1 & -1 & -1 & -1 & 68.1 & 13.00 \\
\hline 3 & -1 & +1 & -1 & -1 & -1 & 47.6 & 12.44 \\
\hline 4 & +1 & +1 & -1 & -1 & +1 & 26.3 & 9.23 \\
\hline 5 & -1 & -1 & +1 & -1 & -1 & 70.7 & 13.18 \\
\hline 6 & +1 & -1 & +1 & -1 & +1 & 54.8 & 13.22 \\
\hline 7 & -1 & +1 & +1 & -1 & +1 & 57.8 & 12.66 \\
\hline 8 & +1 & +1 & +1 & -1 & -1 & 35.9 & 10.93 \\
\hline 9 & -1 & -1 & -1 & +1 & -1 & 43.9 & 11.91 \\
\hline 10 & +1 & -1 & -1 & +1 & +1 & 20.3 & 6.29 \\
\hline 11 & -1 & +1 & -1 & +1 & +1 & 5.4 & 5.84 \\
\hline 12 & +1 & +1 & -1 & +1 & -1 & 2.8 & 5.21 \\
\hline 13 & -1 & -1 & +1 & +1 & +1 & 44.8 & 8.00 \\
\hline 14 & +1 & -1 & +1 & +1 & -1 & 29.3 & 7.61 \\
\hline 15 & -1 & +1 & +1 & +1 & -1 & 16.7 & 5.90 \\
\hline 16 & +1 & +1 & +1 & +1 & +1 & 2.1 & 5.08 \\
\hline 17 & -1 & 0 & 0 & 0 & 0 & 42.3 & 11.81 \\
\hline 18 & +1 & 0 & 0 & 0 & 0 & 26.5 & 6.74 \\
\hline 19 & 0 & -1 & 0 & 0 & 0 & 30.4 & 11.56 \\
\hline 20 & 0 & +1 & 0 & 0 & 0 & 21.3 & 6.45 \\
\hline 21 & 0 & 0 & -1 & 0 & 0 & 24.1 & 9.43 \\
\hline 22 & 0 & 0 & +1 & 0 & 0 & 34.9 & 8.33 \\
\hline 23 & 0 & 0 & 0 & -1 & 0 & 56.4 & 12.85 \\
\hline 24 & 0 & 0 & 0 & +1 & 0 & 20.6 & 6.75 \\
\hline 25 & 0 & 0 & 0 & 0 & -1 & 36.0 & 9.65 \\
\hline 26 & 0 & 0 & 0 & 0 & +1 & 20.3 & 4.64 \\
\hline 27 & 0 & 0 & 0 & 0 & 0 & 37.6 & 7.56 \\
\hline 28 & 0 & 0 & 0 & 0 & 0 & 31.8 & 8.28 \\
\hline 29 & 0 & 0 & 0 & 0 & 0 & 28.3 & 8.43 \\
\hline 30 & 0 & 0 & 0 & 0 & 0 & 29.7 & 7.52 \\
\hline 31 & 0 & 0 & 0 & 0 & 0 & 28.9 & 7.79 \\
\hline
\end{tabular}


Table 3: Criteria for optimizations 1 and 2.

\begin{tabular}{|c|c|c|c|c|}
\hline & Criteria & Target & Min & $\operatorname{Max}$ \\
\hline \multicolumn{5}{|l|}{ Optimization 1} \\
\hline Yield $\left(\eta_{\text {yield }}\right)$ & Target & 20 & 15 & 30 \\
\hline \multirow{6}{*}{$\begin{array}{c}\text { Median size }\left(\eta_{\text {size }}\right) \\
\mathrm{u}_{1} \\
\mathrm{u}_{2}\end{array}$} & Target & 5 & 4.5 & 5.8 \\
\hline & Free & & 35 & 40 \\
\hline & Free & & 2.2 & 3.4 \\
\hline & Free & & 5 & 15 \\
\hline & Constant & 7 & & \\
\hline & Free & & 0 & 100 \\
\hline \multicolumn{5}{|l|}{ Optimization 2} \\
\hline Yield $\left(\eta_{\text {yield }}\right)$ & Target & 20 & 15 & 30 \\
\hline Median size $\left(\eta_{\text {size }}\right)$ & Target & 6 & 5.5 & 6.5 \\
\hline $\mathrm{u}_{1}$ & Free & & 35 & 40 \\
\hline $\mathrm{u}_{2}$ & Free & & 2.2 & 3.4 \\
\hline $\mathrm{u}_{3}$ & Free & & 5 & 15 \\
\hline $\mathrm{u}_{4}$ & Constant & 5 & & \\
\hline $\mathrm{u}_{5}$ & Free & & 0 & 100 \\
\hline
\end{tabular}


Table 4: Sets of conditions proposed for optimizations 1 and 2.

\begin{tabular}{ccccccc}
\hline Optimization & $\mathrm{U}_{1}$ & $\mathrm{U}_{2}$ & $\mathrm{U}_{3}$ & $\mathrm{U}_{4}$ & $\mathrm{U}_{5}$ & $\log (\mathrm{D})$ \\
\hline 1 & 39.9993 & 2.8728 & 14.1457 & 7 & 99.9942 & 0.0205 \\
2 & 39.9914 & 3.1636 & 14.6946 & 5 & 99.3155 & -0.3706 \\
\hline
\end{tabular}


Table 5: Predicted and measured responses for sets of conditions 1 and 2.

\begin{tabular}{ccccc}
\hline & Predicted & Low limit & High limit & Measured \\
\hline yield & & & \\
\hline Optimization 1 & 15.4881 & 10.0454 & 20.9315 & 16.63 \\
Optimization 2 & 16.6481 & 10.6395 & 22.6568 & 15.7 \\
\hline size & & & & \\
\hline Optimization 1 & 5.9059 & 4.6442 & 7.1677 & 4.64 \\
Optimization 2 & 6.3172 & 4.9243 & 7.7101 & 4.40 \\
\hline
\end{tabular}




\section{Captions to figures}

Figure 1: Plot of measured responses versus predicted responses for (a) $\eta_{\text {yield }}$ and (b) $\eta_{\text {size }}$.

Figure 2: The main effects for (a) the yield $\left(\eta_{\text {yield }}\right)$ and (b) the median size $\left(\eta_{\text {size }}\right)$ for a confidence level of 0.90 .

Figure 3: Response-surface plot (upper) and its contour plot of yield $\left(\eta_{\text {yield }}\right)$ : temperature versus duration of hydrolysis with constant level of acid concentration (2.8 M), starch concentration (10 wt $\%$ ) and stirring (50 rpm).

Figure 4: Response-surface plot (upper) and its contour plot of median size $\left(\eta_{\text {size }}\right)$ : temperature versus duration of hydrolysis with constant level of acid concentration $(2.8 \mathrm{M})$, starch concentration (10 wt\%) and stirring (50 rpm).

Figure 5: Response-surface plot (upper) and its contour plot of yield ( $\left.\eta_{\text {yield }}\right)$ : acid concentration versus duration of hydrolysis with constant level of temperature $\left(37.5^{\circ} \mathrm{C}\right)$, starch concentration (10 wt $\%)$ and stirring (50 rpm).

Figure 6: Response-surface plot (upper) and its contour plot of median size ( $\left.\eta_{\text {size }}\right)$ : acid concentration versus duration of hydrolysis with constant level of temperature $\left(37.5^{\circ} \mathrm{C}\right)$, starch concentration (10 wt $\%)$ and stirring (50 rpm).

Figure 7: TEM of negatively stained nanocrystals obtained after $3.16 \mathrm{M} \mathrm{H} \mathrm{H}_{2} \mathrm{SO}_{4}$ hydrolysis of waxy maize starch granules during 5 days, at $40^{\circ} \mathrm{C}, 100 \mathrm{rpm}$ and with a starch concentration of 14.69 wt\%. (a) Aggregates of nanocrystals and (b-d) organizations of nanoplatelets. Scale bar: $50 \mathrm{~nm}$. 
Angellier at al, Figure 1

(a)

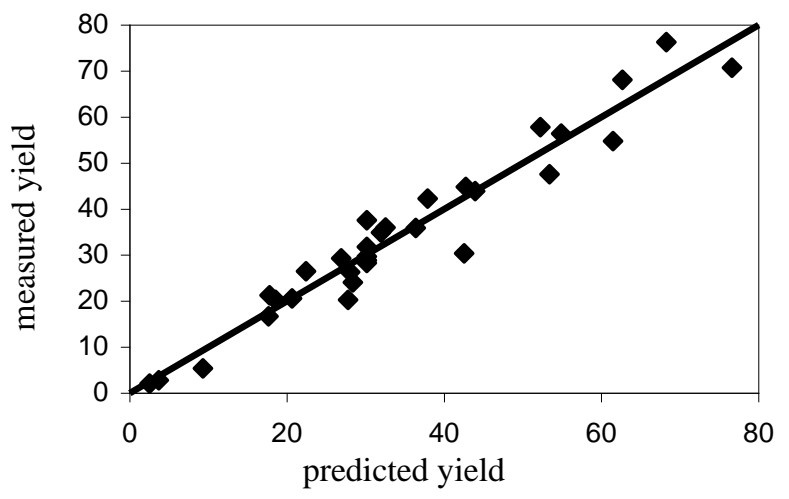

(b)

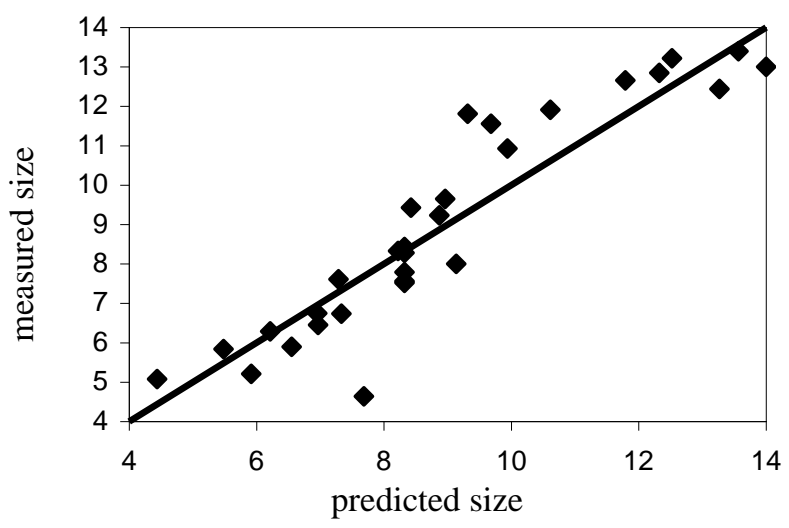


Angellier at al., Figure 2

(a)

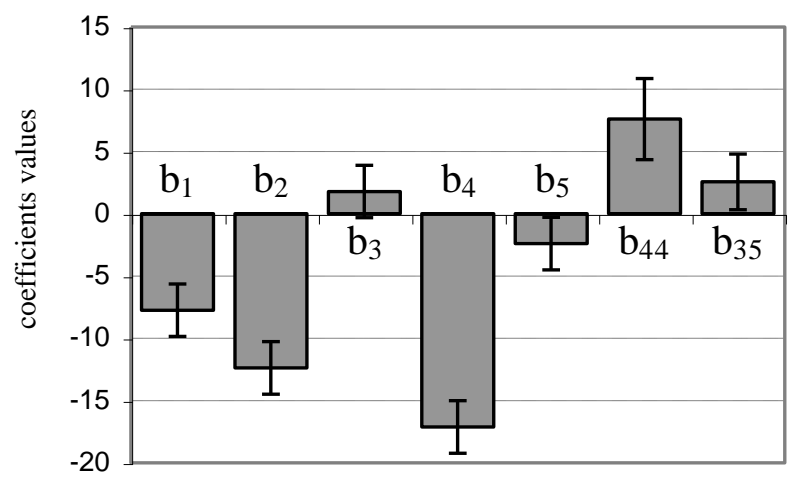

(b)

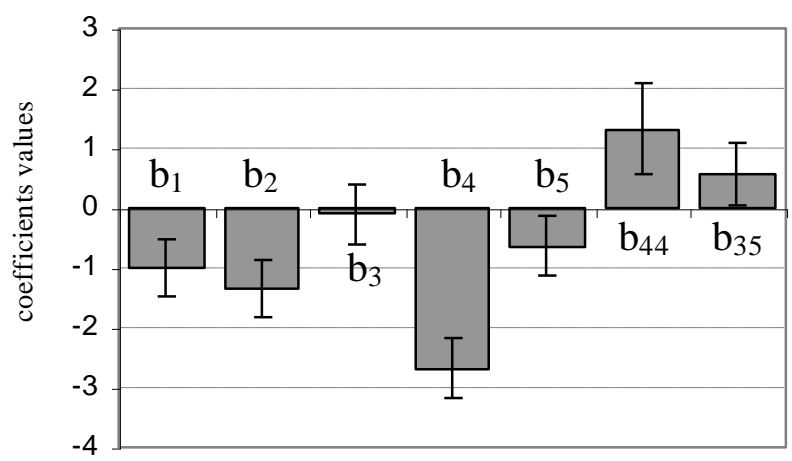


Angellier et al, Figure 3
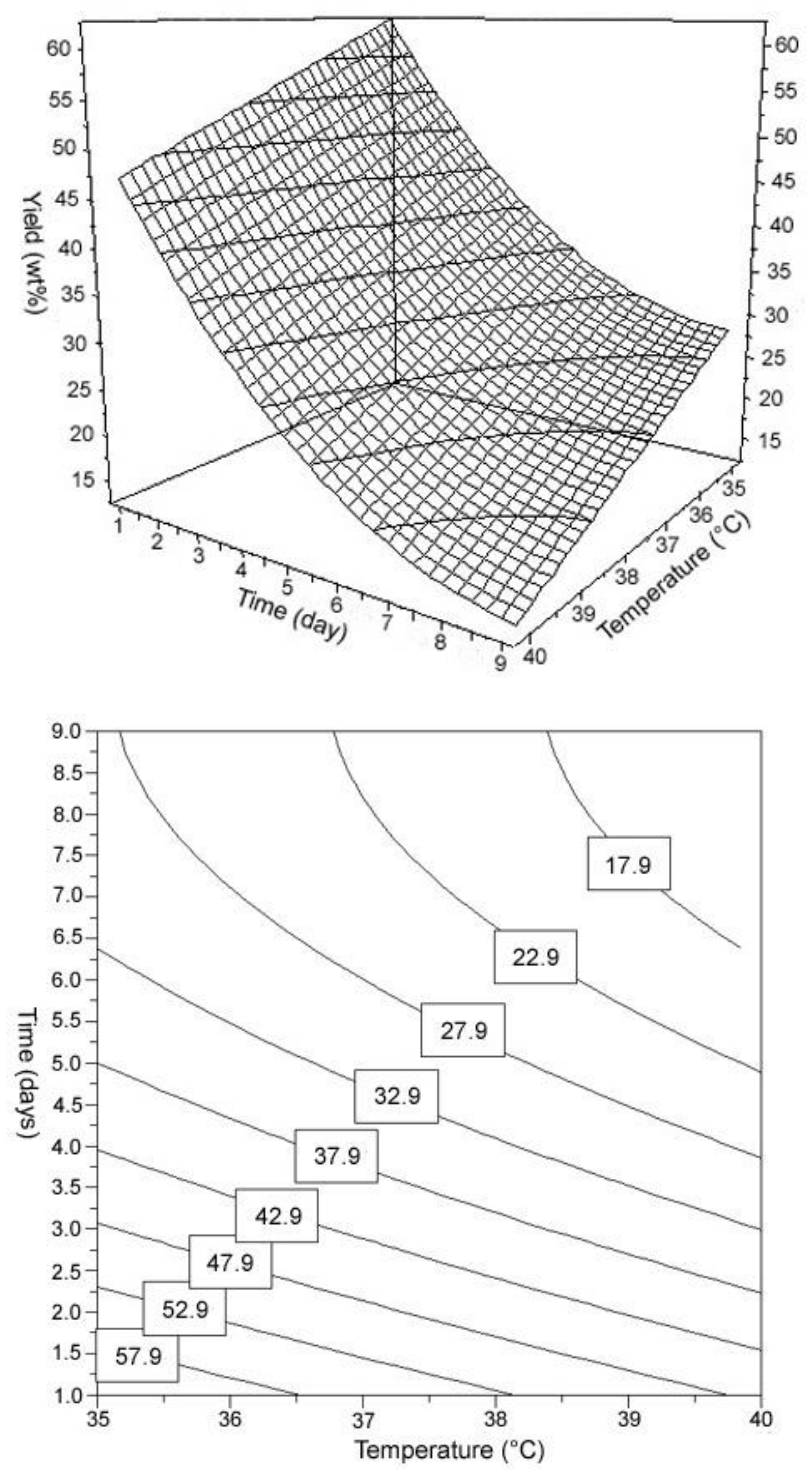
Angellier et al., Figure 4
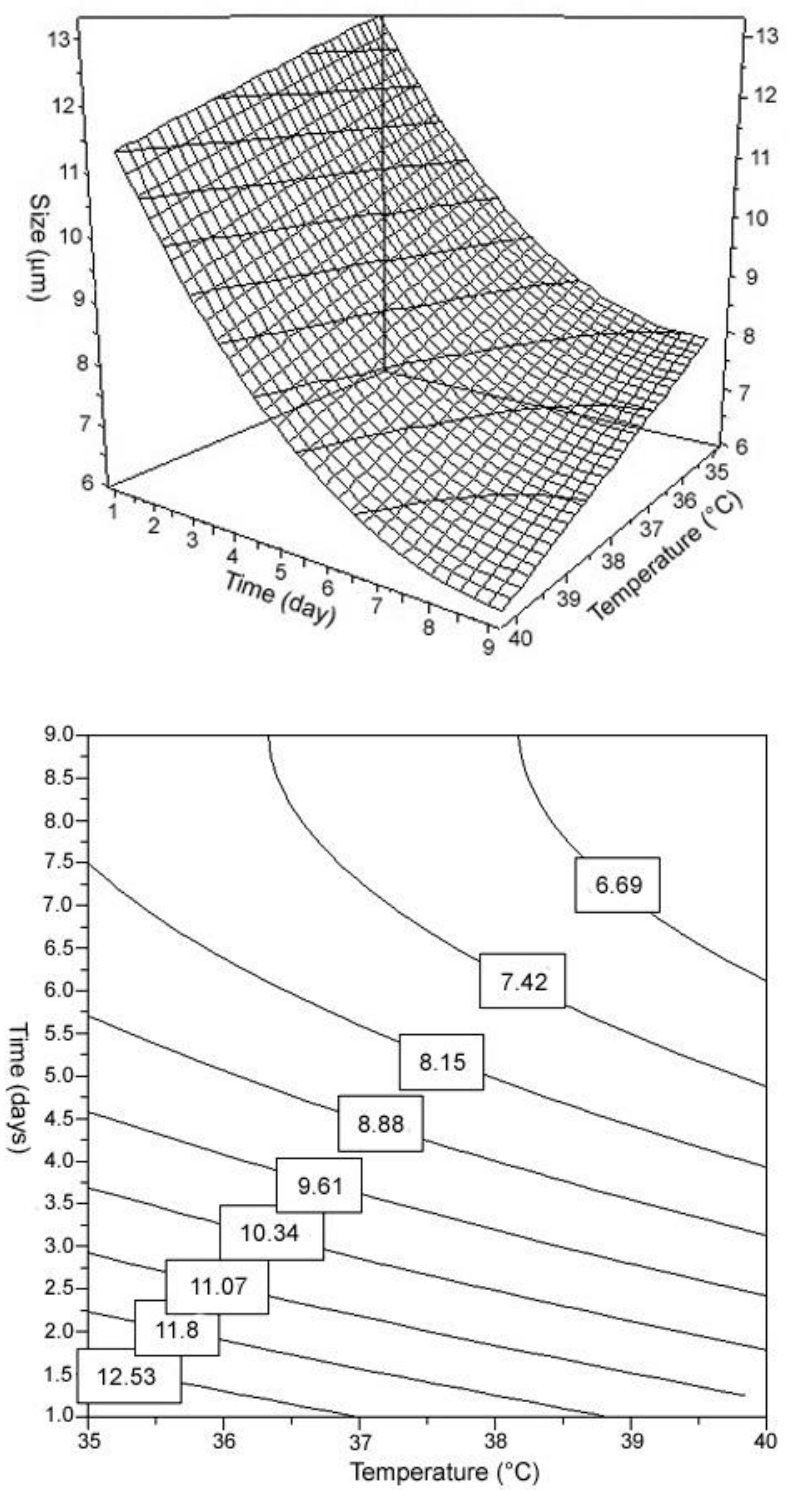
Angellier et al, Figure 5
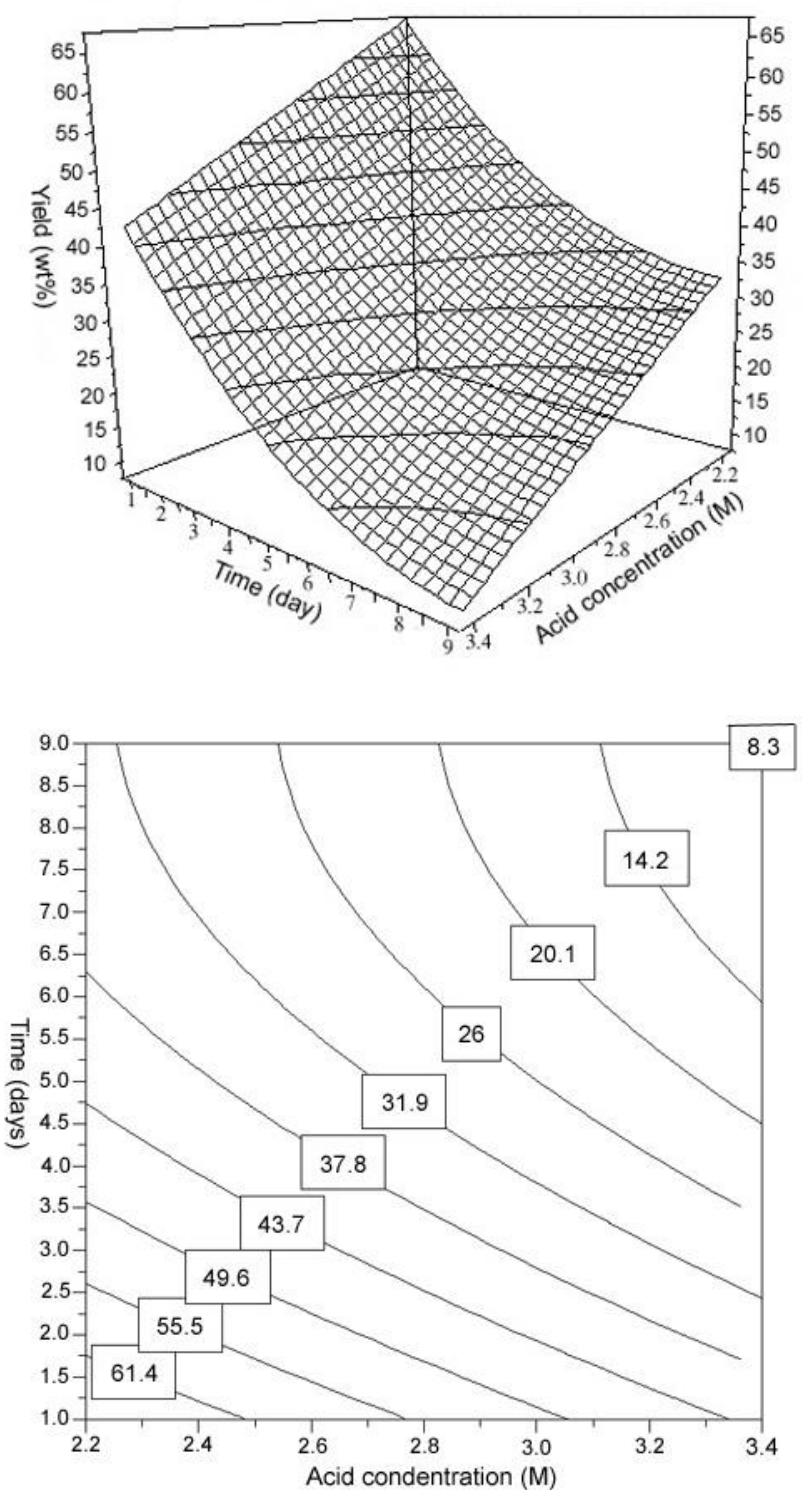
Angellier et al., Figure 6
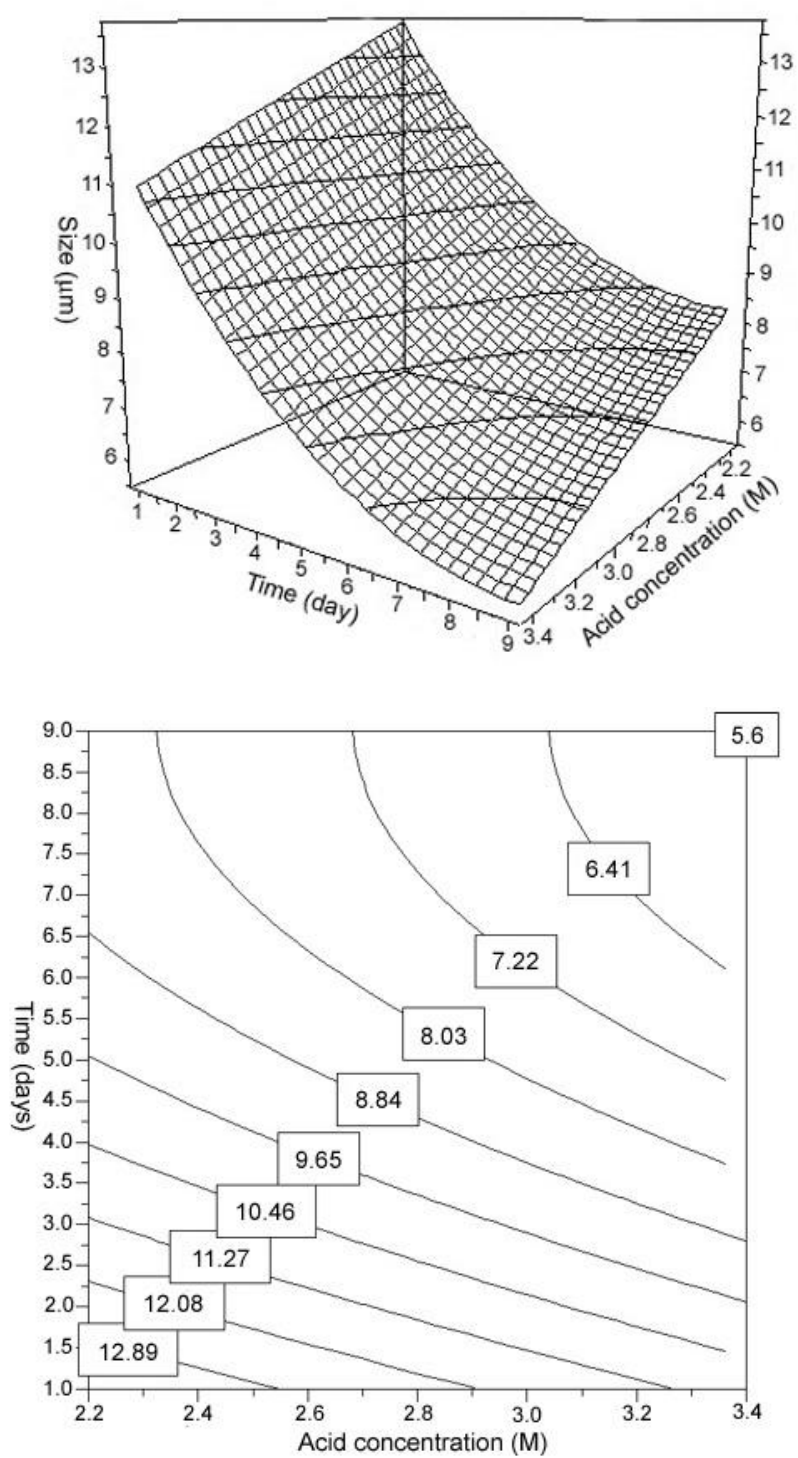
Angellier et al., Figure 7
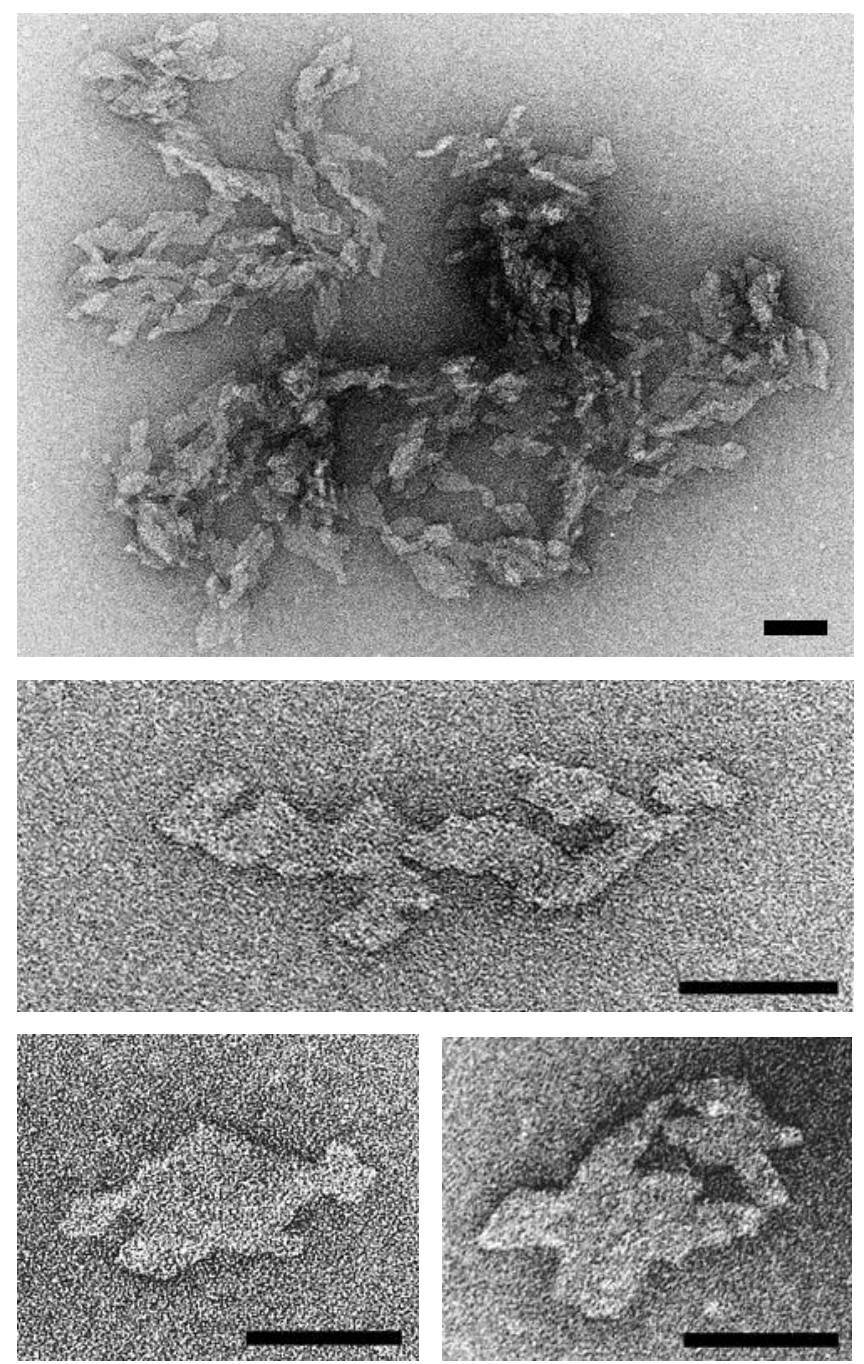